\title{
Instalación para medición de conductividad térmica en composites basados en residuos de biomasa
}

\section{(Device for measuring thermal conductivity of composites based on biomass waste)}

\author{
Luis Velasco Roldán ${ }^{1}$, Leonardo Goyos Pérez ${ }^{1}$, Reinaldo Delgado García ${ }^{1}$, Luis Freire Amores ${ }^{2}$
}

\begin{abstract}
Resumen:
Se ha diseñado, construido y calibrado un banco de pruebas normalizado para la determinación de la conductividad térmica de materiales aislantes de construcción. El aparato, sencillo y económico, pretende convertirse en herramienta replicable y útil para el desarrollo de múltiples investigaciones en torno a materiales basados en residuos o recursos no valorizados para la producción de aislamientos térmicos baratos de producción local no industrializados que repercutan en la mejora de la eficiencia energética de las edificaciones. La principal aportación del banco de pruebas es la posibilidad del análisis de aislamientos compuestos de mayor espesor y distintos formatos gracias al diseño de la prensa, que permite el ajuste y la presión de las placas sobre las muestras, sosteniendo estas en el aire y evitando cualquier transmisión por conducción no deseada.
\end{abstract}

Palabras clave: eficiencia energética; aislamiento térmico; residuos vegetales

\begin{abstract}
:
A standardized test bench has been designed, built and calibrated to determine the thermal conductivity of insulating building materials. The device, simple in design and economical, aims to become a replicable and useful tool for the development of multiple research on innovative materials based on waste or unvalued resources for the production of non-industrial and locally produced cheap thermal insulating materials which lead to the improvement of buildings energy efficiency. The main contribution of the test bench is the possibility of analyzing insulation compounds with more thickness and different formats thanks to the press design, which allows the setting and the pressure of the plates on the samples, holding these in the air and preventing any transmission by unwanted conduction.
\end{abstract}

Keywords: thermal conductivity; thermal insulation; vegetable waste

\footnotetext{
${ }^{1}$ Universidad de las Fuerzas Armadas, Sangolquí - Ecuador, (luisvelascoroldan@espe.edu.ec)

2Universidad Tecnológica Equinoccial, Quito - Ecuador, (luis.freire@ute.edu.ec)
} 


\section{Introducción}

La falta de consideración de la variabilidad climática en Latino América, así como la debilidad en un desarrollo industrial que pueda correr paralelo a una investigación tecnológica y constructiva, viene traduciéndose en soluciones edificatorias determinadas únicamente por los productos industriales disponibles o importables, generando una profunda desorientación edificatoria. De mantenerse esta dinámica, carente de investigaciones que recuperen, profundicen y evolucionen los conocimientos constructivos ancestrales, se augura una preocupante inadaptación tecnológica, constructiva y ambiental que generará un desarrollo edificatorio claramente insostenible. Es por ello necesario la evolución de los modelos bajo un nuevo prisma de sostenibilidad energética, social y económica.

Las dinámicas constructivas vienen desde principio de siglo $\mathrm{XX}$ sustituyendo en los países industrializados los muros tradicionales de gran espesor y un solo material (tierra, piedra, ladrillo, etc.) por muros heterogéneos compuestos de diversas capas, donde cada una de ellas cumple específicamente con alguno de los requerimientos del muro (estanqueidad, resistencia, aislamiento térmico, etc.). Esta estrategia fomenta la sustitución de materiales polivalentes por materiales compuestos de altísimo rendimiento frente a la función encomendada. Dicha lógica viene apoyada por la necesidad de reducir al máximo el espesor de los muros frente al elevado valor del suelo y la aplicación de cada vez más estrictas normativas térmicas, acústicas, estructurales, etc. Un vistazo a la situación global de América Latina, África o Asia, nos muestra realidades muy distintas en donde dicho modelo constructivo no tiene sentido alguno ante realidades industriales en proceso de desarrollo y grandes extensiones de reducida presión demográfica. En gran parte de dichas áreas la dinámica constructiva ancestral continua teniendo vigencia. Los muros homogéneos de gran espesor formados por materiales que incorporen desechos agrícolas o fibras vegetales locales como materia prima de aislamiento térmico tienen un campo de desarrollo real y útil dentro de un deseable desarrollo sostenible.

Es por todo ello que se requiere caracterizar los diferentes compuestos de fabricación local. Con este objetivo se realizó el diseño del banco de pruebas de transferencia de calor de tipo placa caliente (Method, 2013b) específico para investigaciones en torno a dichos compuestos de fabricación local, buenas prestaciones térmicas y de resistencia (en el caso de ser esta necesaria), bajo coste y reducido impacto ecológico. En la actualidad, marzo de 2015, no existe en Ecuador un aparato de medición de tales características ni material alguno que cuente con un coeficiente de conductividad certificado. El aparato se ha construido siguiendo el método de ensayo para la determinación de las propiedades de transmisión térmica en estado estacionario de muestras de aislamiento térmico con un calentador delgado de densidad de potencia uniforme con bajo flujo de calor lateral según criterios normativos de la ASTM (Method, 2013b), (Method, 
2014). La norma establece criterios de laboratorio para la medida del flujo unidireccional y estacionario de calor a través de dos muestras planas y homogéneas de caras paralelas y espesor conocido testadas a temperaturas constantes mediante un sistema de placa caliente central y doble placa fría exterior. Del ensayo se determina mediante la ley de Fourier la conductividad térmica.

$Q=A K(-d T / d X)$

Donde:

Q: flujo de calor a lo largo del espesor, medida en W, A: área de la sección del material, perpendicular al flujo de calor, medida en $\mathrm{m}^{2}, \mathrm{dT} / \mathrm{dx}$ : es el gradiente de temperatura entre la placa fría y caliente, medida en ${ }^{\circ} \mathrm{K}$ siendo $\mathrm{dx}$ el espesor de de la muestra, $\mathrm{k}$ : coeficiente de conductividad térmica del material, medida en $\mathrm{W} \mathrm{m}^{-1} \mathrm{~K}^{-1}$

\section{Metodología}

El banco de pruebas de medición de conductividad se fabrica siguiendo las pautas, prescripciones y recomendaciones de las normas ASTM C177-13 (Method, 2013b), ASTM C1114-13 (Method, 2013a) , ASTM C168 - 13 (Method, 2014) y ASTM C518-10 (Method, 2013c).

Dichas normas no describen un diseño concreto, sino que describen los requerimientos mínimos necesarios para la construcción de un modelo que asegure una transmisión estable y constante de la energía estrictamente por conducción de forma perpendicular a los focos frío y caliente; unas sondas de control sobre el funcionamiento del sistema y una medición precisa del flujo de calor y las temperaturas resultantes del experimento. Dicho método, prescriptivo, aporta la suficiente flexibilidad al modelo como para permitir la construcción de aparatos de ensayo destinados al análisis de compuestos, con un rango de características y formatos muy amplio (Milagrosa, Sánchez, Centeno, \& Lazcano, 2002), (Martínez Fuentes, n.d.), (J. Diez Campos, E. García Breijo. I Perez Clemente, J.V. Ros Lis. J. Soto Camino, 2007), (Lopez et al., 2000).

\section{Resultados}

La Figura 1 muestra la concepción general del banco de prueba. El mismo está compuesto por un bastidor con un soporte fijo y otro ajustable, los cuales sostienen las placas frías (focos fríos), entre las cuales se sitúan las muestras a ensayar y entre ellas el elemento calefactor (foco caliente). La instalación cuenta con una campana de vacío para realizar el ensayo bajo estas condiciones, de ser necesario. 

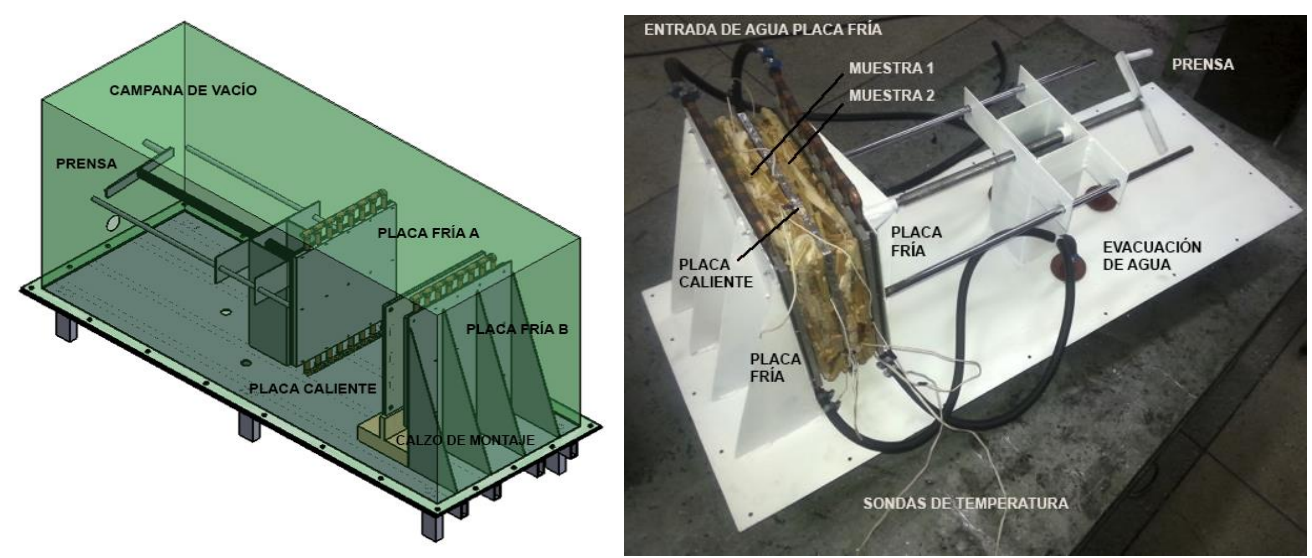

Figura 1. Esquema y fotografía de aparato de medición

\section{Foco caliente}

En la Figura 2 puede observarse el elemento calefactor regulado (Placa caliente) y el anillo perimetral de guarda. La placa caliente se construye mediante cinta de aleación de Ni80Cr20 (nicrom) en soporte de mica. El elemento resistivo se encuentra aislado mediante láminas de mica y forman a su vez un emparedado entre láminas de aluminio de espesor 0,2 mm. La distribución del elemento resistivo es uniforme en toda el área de calentamiento (150 x $150 \mathrm{~mm}$ ) y el espesor de las placas externas de aluminio asegura una alta conductividad y baja inercia térmica en ambas caras de trabajo. El voltaje aplicado a la placa caliente $(0.1-18 \mathrm{v}$ de corriente continua) es regulado mediante un potenciómetro para fijar la temperatura y por ende el flujo de calor. La selección del voltaje más adecuado será en función de la muestra ensayada en la búsqueda de una diferencia de temperatura de $20^{\circ} \mathrm{C}$, al ser este el patrón de temperaturas más habitual de medida en materiales de construcción

\section{Anillo perimetral}

Un anillo perimetral de 300 × $300 \mathrm{~mm}$ en su parte exterior (anillo de guarda) rodea la placa caliente, separado $3 \mathrm{~mm}$ de esta mediante material aislante. Al igual que la placa caliente, este anillo contiene una resistencia eléctrica aislada por mica y protegida por placas de aluminio, en este caso de $2 \mathrm{~mm}$ de espesor. El sistema de control del equipo garantiza que la guarda mantenga la misma temperatura que la placa caliente, con el fin de evitar flujo de calor en sentido radial. La diferencia de temperatura entre la guarda y la fuente primaria de energía tal y como determina la norma (Method, 2013b), no ha superado durante las pruebas los $0,2^{\circ} \mathrm{C}$, asegurándose una transmisión lineal e unidireccional de la energía entre la placa caliente y las placas frías. 


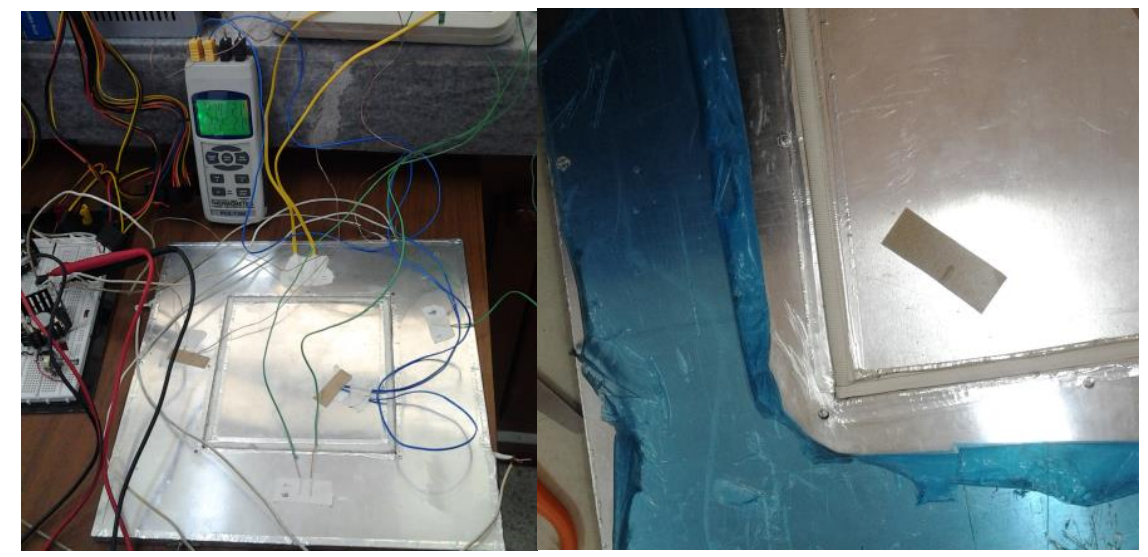

Figura 2. Placa caliente - guarda perimetral y la separación entre ambos elementos calefactores.

\section{Placa fría}

Las placas frías (Figura 3) tienen la función de disipar la energía que llega desde la placa caliente a través de las muestras que se desea medir. Están formadas por dos planchas de aluminio de $30 \times 30 \mathrm{~cm}$ y $10 \mathrm{~mm}$ de espesor con ocho ranuras de $6 \times 6 \mathrm{~mm}$ en su cara interior destinadas a albergar una serie de conducciones de cobre de $8 \mathrm{~mm}$ por las que circula agua. Este recorrido a través del interior de las placas se realiza mediante colectores para asegurar la imprescindible uniformidad de la temperatura en toda la placa. De haberse realizado la distribución de agua mediante un serpentín, el progresivo calentamiento del agua en su recorrido a través de la placa hubiera generado distintas temperaturas sobre esta distorsionando la linealidad del flujo de calor. Para la correcta transmisión del flujo de energía se garantiza el mayor contacto posible entre los elementos refrigeradores y las placas. El espesor de las placas mejora la uniformidad de la disipación de la energía.

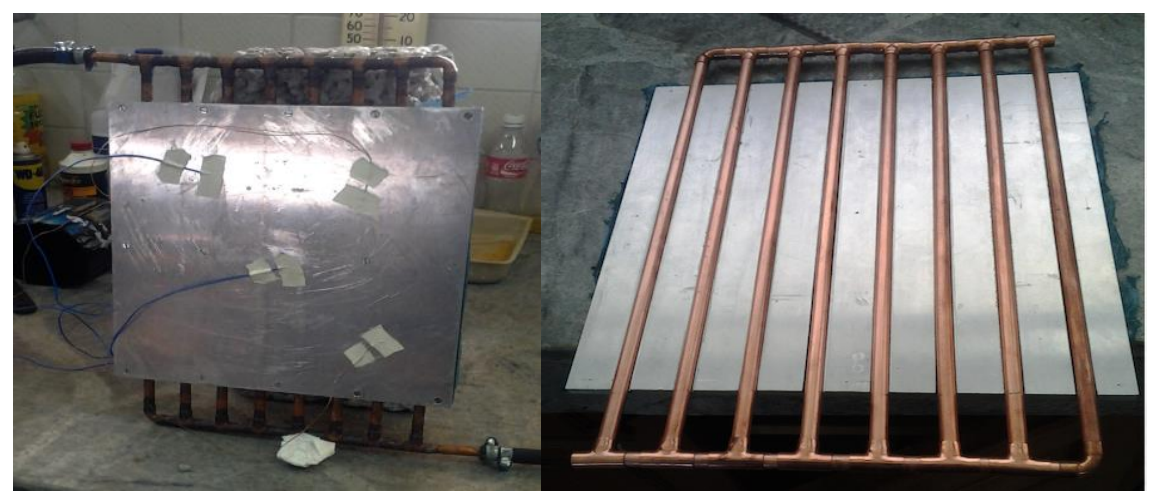

Figura 3. Fotografía de la placa fría y su sistema de distribución de agua.

\section{Prensa}

La prensa es la encargada de comprimir el conjunto asegurando un buen contacto entre placas y muestras sin forzar una compresión excesiva que pueda deformar las muestras y variar la densidad de, por ejemplo, los materiales fibrosos. Un aislamiento térmico envuelve las placas y la 
muestra para evitar pérdidas de calor por el perímetro en muestras de gran espesor (Method, 2013b)

\section{Campana de vacío}

Adicionalmente, con la pretensión de realizar pruebas en ausencia total de convección, se ha construido una campana de acero de $1 \mathrm{~mm}$ de espesor que envuelve el aparato de medición y permite la creación del vacío en su interior. De esta forma será posible en futuras pruebas eliminar toda transmisión de energía por convección entre el perímetro de la muestra y su entorno. La experimentación en vacío permitirá calibrar la influencia de las pérdidas por convección en los experimentos de conductividad térmica a grandes altitudes (Sangolquí se encuentra a 2400 metros sobre el nivel del mar). Con ella será posible determinar el porcentaje de dispersión que resulte de la experimentación que prescinda de evaluar dichas pérdidas por convección. Dicha investigación fue iniciada por Raimundo López con una campana de similares características en la Universidad Autónoma de México DF (Lopez et al., 2000)

\section{Sondas de control y registro}

Se ha dispuesto un total de 12 sondas de temperatura tipo $\mathrm{K}$ (cromel-alumel) con dirección física (MAC) conectadas a los circuitos de control. Los valores de temperatura pueden ser monitoreados por medio de tres termómetros digitales.

Placa caliente: Dos termopares tipo $\mathrm{K}$ en cada una de las caras de la placa caliente para verificar la igualdad de temperaturas en ambas caras y uniformidad de temperaturas a largo de la placa. Anillo perimetral: 4 termopares distribuidos en el perímetro.

Placas frías: dos termopares por cada cara en contacto con la muestra, uno en el centro de la placa (zona correspondiente a la posición de la placa caliente y el otro en la zona exterior (zona correspondiente a la posición de la guarda). Se controla permanentemente la diferencia de temperaturas entre la placa caliente y la guarda mediante el sistema de control

\section{Sistema de control electrónico}

El sistema de control (figuras 4 y 5) pretende el establecimiento, de forma automática de idénticas temperaturas entre la placa caliente y la placa perimetral para asegurar un flujo de calor perpendicular a las muestras a ensayar. El sistema de control cuenta con una doble fuente de alimentación. La fuente de 5VDC, alimenta el micro-controlador y los sensores de temperatura conectada a un transformador 120V-12VAC. Cuenta con sistema de protección con fusible de 1A, 
rectificado mediante puente de diodos, además de un filtrado de señal mediante capacitor de 1000 uF, lo cual garantiza una señal constante y sin ruido. La fuente de 18VDC, alimenta las placas interna y externa y posee las mismas características de la fuente de 5VDC. En este caso el fusible que es de $2 \mathrm{~A}$.

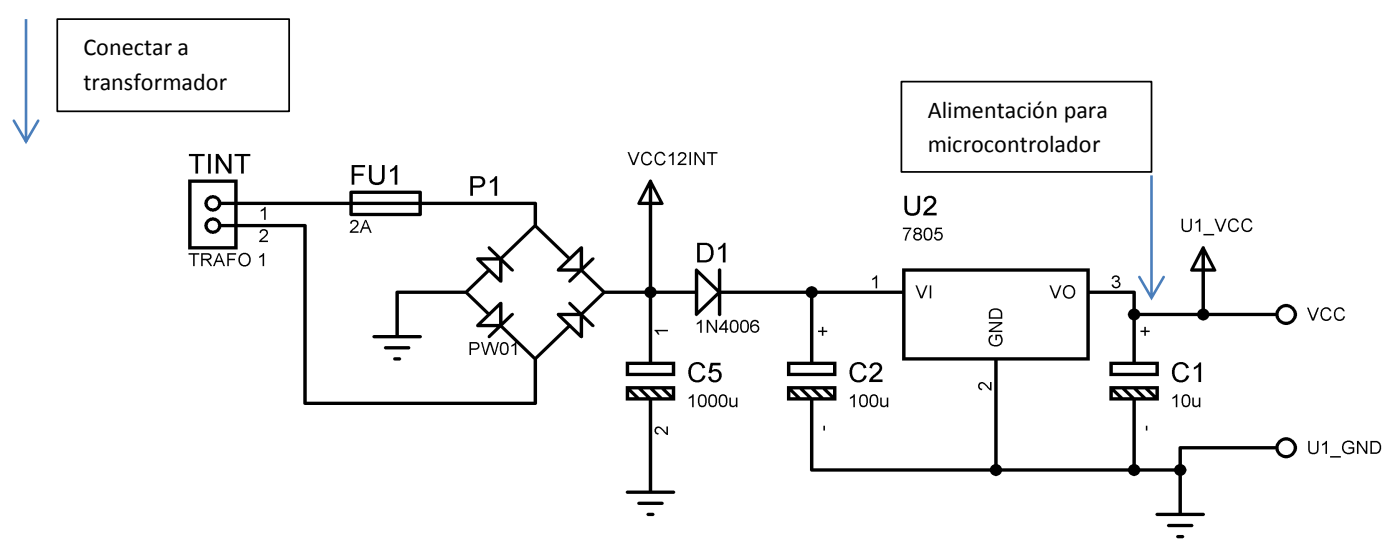

Figura 4. Sistema de control de la placa interna. (TINT - Transformador 110V-12V (Corriente Alterna), FU1 - Fusible de 1A, P1 - Puente rectificador de diodos, D1 - Diodo para protección anticorriente, U2 - Regulador de voltaje de 5V, C1, C2, C5 - Capacitores de 10uF, 100uF y 1000uF respectivamente)

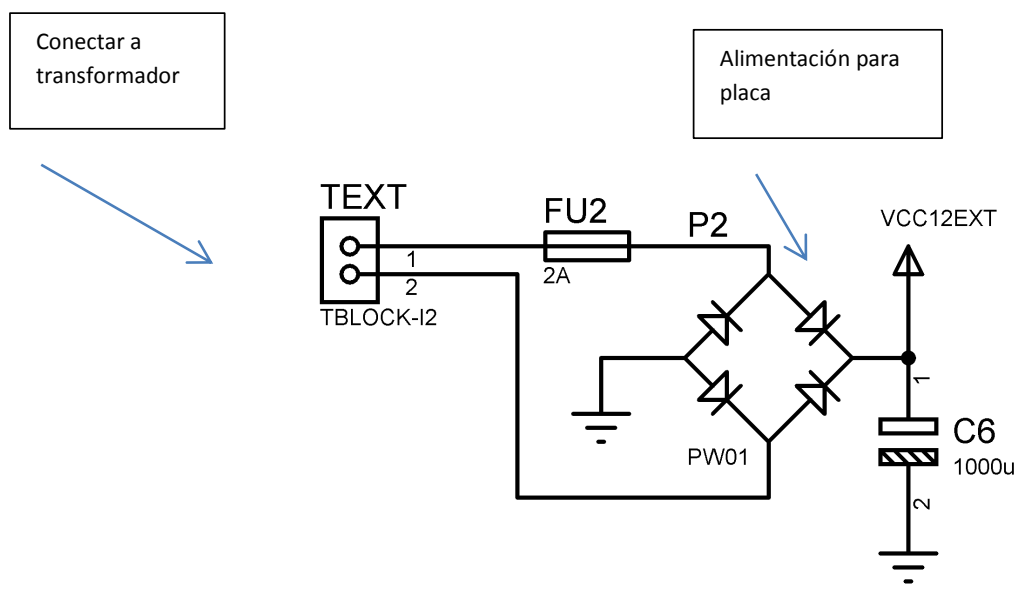

Figura 5. Sistema de control de la placa perimetral (TEXT - Transformador 110V-24V (Corriente Alterna), FU2 - Fusible de 2a , P2 - Puente rectificador de diodo, C6 - Capacitores de 1000uF)

\section{Sistema de Control. Placa caliente}

Se ha utilizado un sistema de control en lazo abierto, tal como se ilustra en la Figura 6. El potenciómetro regula el voltaje de alimentación suministrado a la placa caliente. El microcontrolador lee la señal analógica procedente del potenciómetro y envía una señal de control hacía la base del transistor y finalmente se aplica el voltaje especificado a la placa. El voltaje mínimo suministrado es de $0 \mathrm{~V}$ y el máximo es de $18 \mathrm{~V}$. 


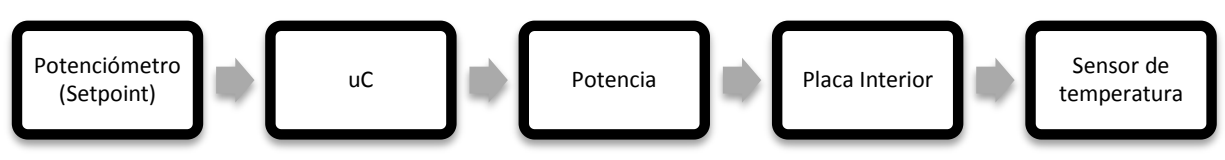

Figura 6. Esquema de funcionamiento placa caliente ( $\mathrm{UC}$ - Microcontrolador, Potencia - PWM (regulación de voltaje por ancho de pulso) y transistor, Sensor de temperatura - Sensor Dallas DS18B20)

El potenciómetro regula el voltaje de alimentación suministrado a la placa caliente. El microcontrolador lee la señal analógica procedente del potenciómetro y envía una señal de control hacía la base del transistor y finalmente se aplica el voltaje especificado a la placa (Figura 7). El voltaje mínimo suministrado es de $0 \mathrm{~V}$ y el máximo es de $18 \mathrm{~V}$.

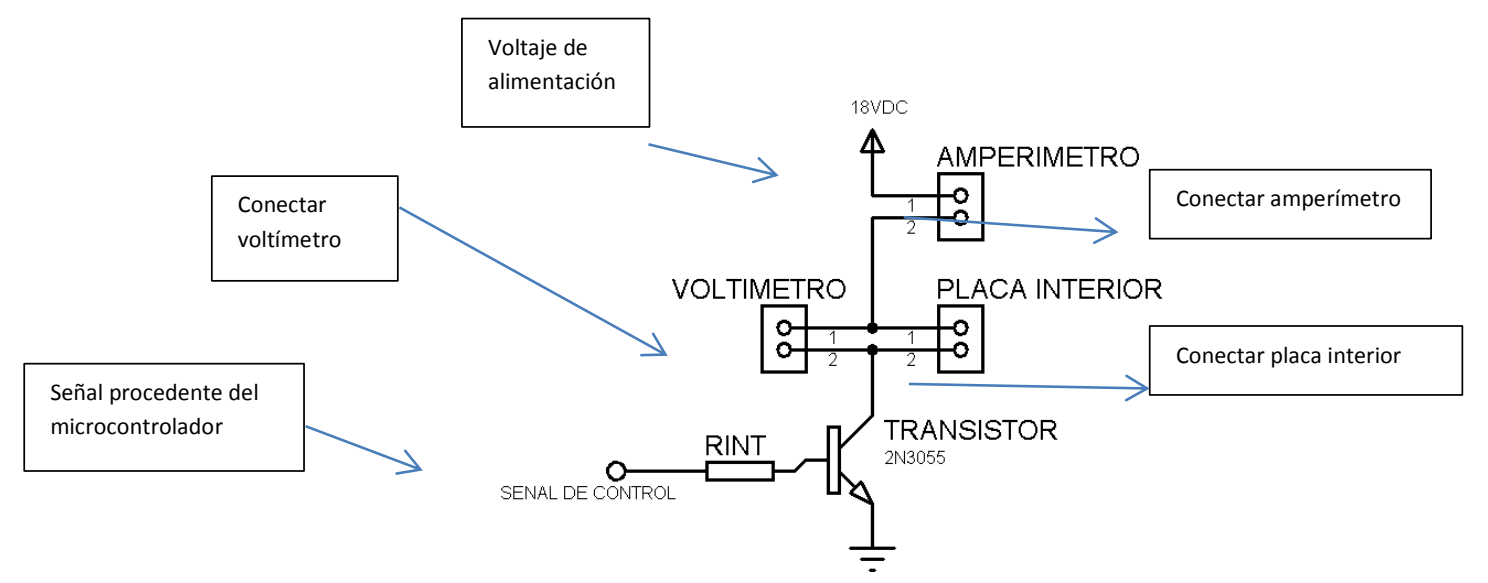

Figura 7. Esquema de potencia placa interior (RINT - Resistencia de base para transistor de 100 ohm)

Sistema de Control. Guarda perimetral. El sistema de control empleado es un lazo cerrado con un controlador PID, tal como se describe en la Figura 8.

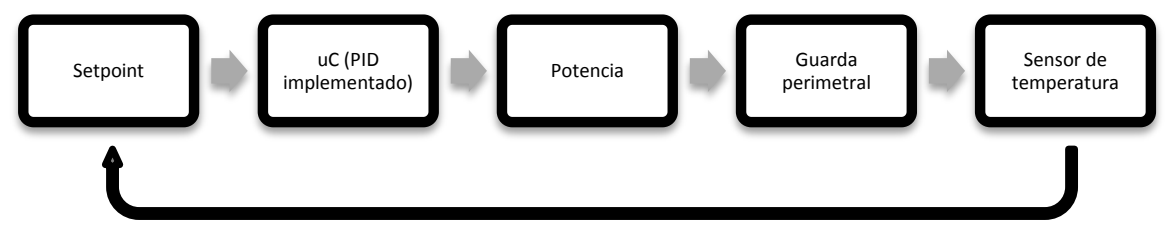

Figura 8. Esquema del de funcionamiento guarda perimetral (Setpoint - Valor de temperatura que se pretende alcanzar, uC - Microcontrolador, PID -Proporcional, Integral y Derivativo. Técnica de sistemas de control aplicada para el control de una variable, en este caso, la temperatura de la placa, Potencia - PWM (regulación de voltaje por ancho de pulso) y transistor, Sensor de temperatura - Sensor Dallas DS18B20)

Se hace necesario un control para mantener las placas a la misma temperatura. La información del Setpoint es suministrada por el sensor de temperatura de la placa interior. El controlador PID se imprementa mediante la programación del micro-controlador. Los cálculos realizados por el controlador son transformados en una señal de control enviada hacia el transistor. Finalmente el 
lazo de control se complementa con la retroalimentación dada por el sensor de temperatura DS18B20 (Figura 9).

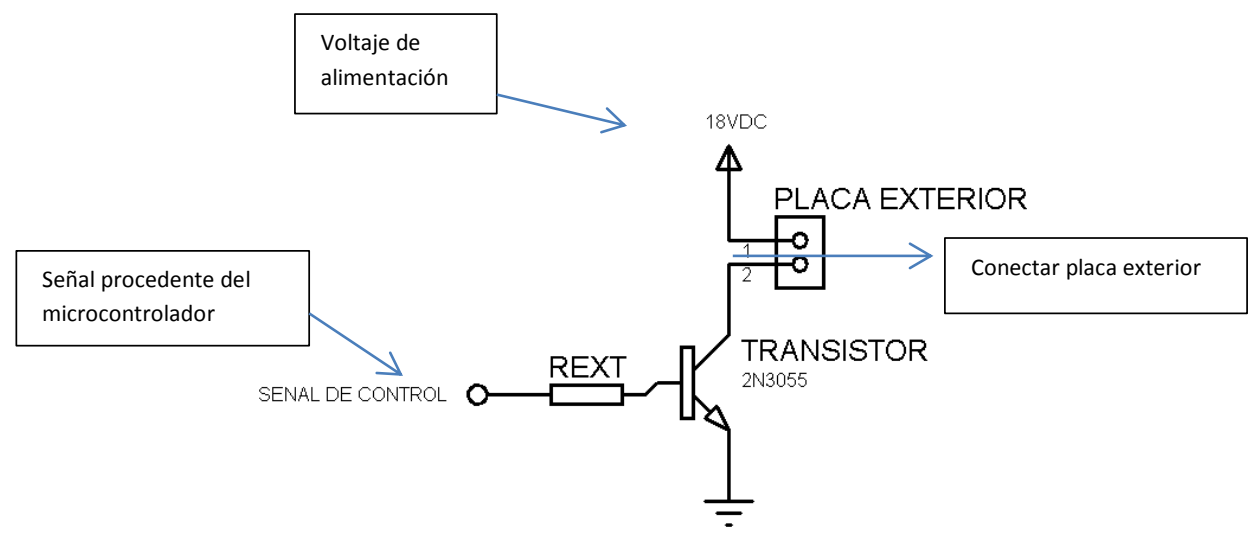

Figura 9. Esquema de potencia placa exterior (REXT - Resistencia de base para transistor de 110 ohm)

\section{Obtención de la Función de Transferencia}

Se aplicó un voltaje constante de $12 \mathrm{~V}$ en la placa interior mientras la temperatura se estabiliza. Los datos experimentales se tomaron con un intervalo de 60 segundos, lo cual se considera suficiente teniendo en cuenta que el tiempo de estabilización de la placa es de aproximadamente 4 horas.

Una vez obtenidos los datos experimentales de temperatura, se procede a realizar el modelamiento mediante la ayuda del programa Matlab, el cual permite obtener una función de transferencia que se ajuste a las curvas de los datos obtenidos de las mediciones mediante la herramienta Ident.

La aproximación con la función de transferencia obtenida es del 90\% lo cual es una suficiente para proseguir con el cálculo del controlador PID.

El sistema de control resultante se describe en la Figura 10 en conjunto con la Tabla 1.

\section{Discusión}

La calibración del aparato se realizó, tal y como dicta la norma ASTM C177-13 (Method, 2013b) mediante el ensayo de dos muestras de materiales de conductividad conocida, ensayada y certificada por laboratorios homologados a nivel nacional, en este caso por las marcas Knauf $\mathrm{GmbH}$ y Webber siguiendo protocolos de la norma UNE EN ISO 10456. Se realizaron en concreto 5 ensayos con dos muestras de placa de yeso laminado de la marca Knauff de $15 \mathrm{~mm}$ de espesor, una densidad de $728 \mathrm{~kg} / \mathrm{m} 3$ y una conductividad térmica de $0,25 \mathrm{~W} / \mathrm{m}^{2}{ }^{\circ} \mathrm{K}$ para un diferencial de temperatura de $20^{\circ} \mathrm{C}$ y 5 ensayos con dos placas de poliestireno expandido de densidad $25 \mathrm{~kg} / \mathrm{m}^{3}$ de $0,03 \mathrm{~W} / \mathrm{m}^{2}$ o $\mathrm{K}$ para un diferencial de temperatura de $20^{\circ} \mathrm{C}$ de densidad de 
$15 \mathrm{~mm}$ de espesor de la marca Webber. Todos los ensayos se realizaron con las muestras estabilizadas en su contenido de humedad a $65 \%$, y los resultados se presentan en la Tabla 2.

Se determina por lo tanto que el aparato ofrece una precisión de $\pm 6,6 \%$ para un diferencial de temperaturas de $20^{\circ} \mathrm{C}$

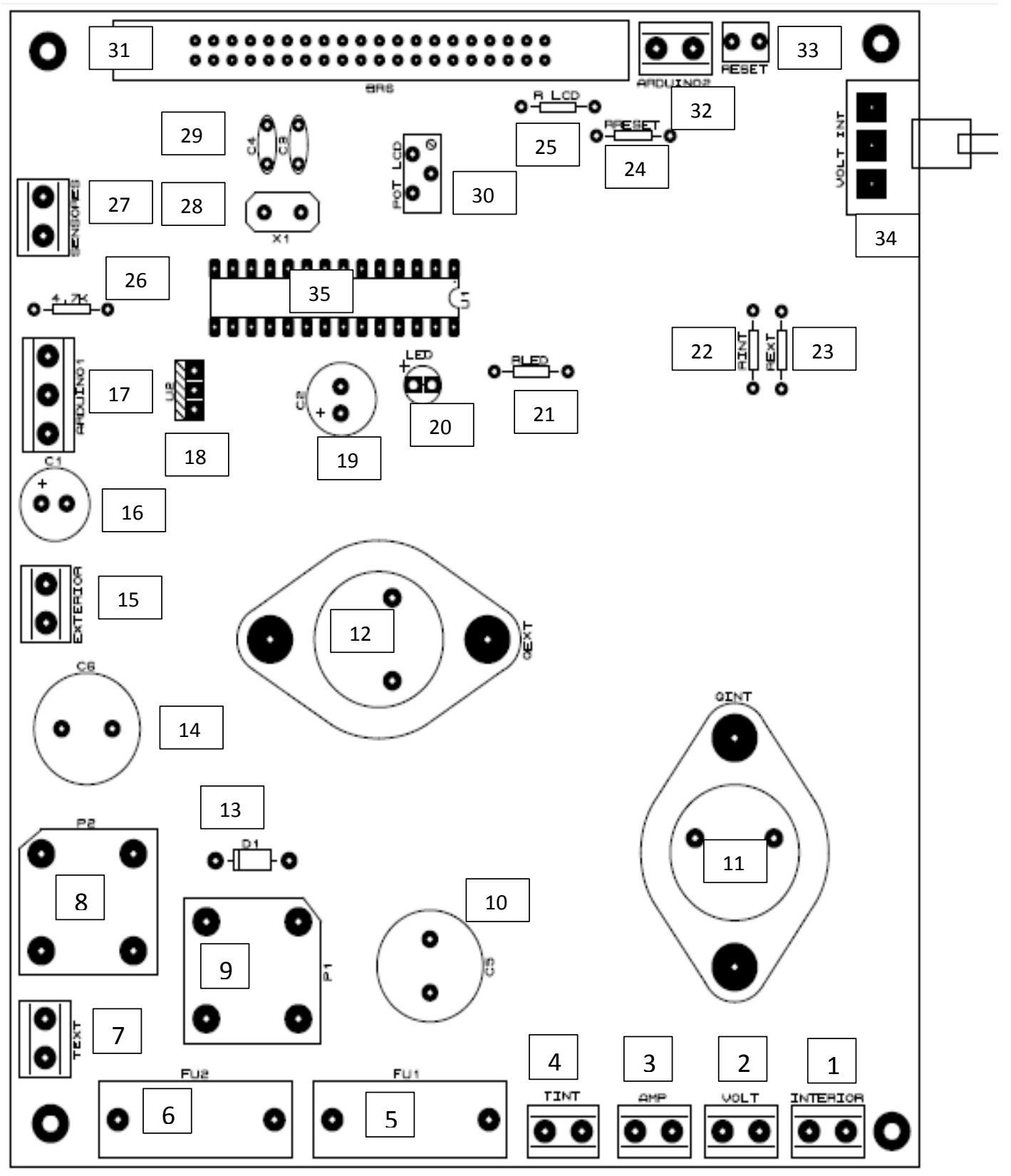

Figura 10. Sistema de control ( AMP: Conectar Multímetro en modo de medidor de corriente contínua (escala 200mA); VOLT: Conectar Multímetro en modo de medidor de voltaje continuo (escala 200 VDC) ). 
Tabla 1. Elementos del sistema de control (acorde a la Figura 10)

\begin{tabular}{|c|c|l|}
\hline Número & Nombre & \\
\hline 1 & INTERIOR & Bornera para conexión de placa interior \\
\hline 2 & VOLT & Bornera para conexión de voltímetro \\
\hline 3 & AMP & Bornera para conexión de amperímetro \\
\hline 4 & TINT & Bornera para conexión de transformador 110V/24V \\
\hline 5 & FU1 & Fusible 1A \\
\hline 6 & FU2 & Fusible 2A \\
\hline 7 & TEXT & Bornera para conexión de transformador 110V/12V \\
\hline 8 & P2 & Puente rectificador \\
\hline 9 & P1 & Puente rectificador \\
\hline 10 & C5 & Capacitor electrolítico \\
\hline 11 & QINT & Transistor para placa interior \\
\hline 12 & QEXT & Transistor para placa interior \\
\hline 13 & D1 & Diodo anticorriente \\
\hline 14 & C6 & Capacitor electrolítico \\
\hline 15 & EXTERIOR & Bornera para conexión de placa exterior \\
\hline 16 & C1 & Capacitor electrolítico \\
\hline 17 & ARDUINO1 & Bornera para conexión de pines de arduino \\
\hline 18 & U2 & Regulador de voltaje 5V \\
\hline 19 & C2 & Capacitor electrolítico \\
\hline 20 & LED & LED indicador \\
\hline 21 & RLED & Resistencia \\
\hline 22 & RINT & Resistencia \\
\hline 23 & REXT & Resistencia \\
\hline 24 & RRESET & Resistencia \\
\hline 25 & RLCD & Resistencia \\
\hline 26 & $4.7 K$ & Resistencia \\
\hline 27 & SENSORES & Bornera para conectar sensores DS18B20 \\
\hline 28 & X1 & Cristal de oscilación 8MHz \\
\hline 29 & C3,C4 & Capacitores cerámicos \\
\hline 30 & POT LCD & Potenciómetro para regular luz de display \\
\hline 31 & BR6 & Bornera para conectar display \\
\hline 32 & ARDUINO2 & Bornera para conexión de pines de arduino \\
\hline 33 & RESET & Pulsador de reset para microcontrolador \\
\hline 34 & VOLT INT & Potenciómetro \\
\hline 35 & U1 & Microcontrolador \\
\hline & & \\
\hline 15 & & \\
\hline & &
\end{tabular}

Tabla 2. Resultados de ensayos de calibración

\begin{tabular}{|c|c|c|c|c|c|c|c|c|c|c|}
\hline \multirow{3}{*}{ MATERIAL } & \multirow{3}{*}{$\begin{array}{c}N^{0} \\
\text { PRUEBA }\end{array}$} & \multirow{3}{*}{$\begin{array}{l}\mathrm{T} 2 \\
{ }^{\circ} \mathrm{C}\end{array}$} & \multirow{3}{*}{\begin{tabular}{|l|}
$\mathrm{T} 3$ \\
${ }^{\circ} \mathrm{C}$
\end{tabular}} & \multirow{3}{*}{$\begin{array}{l}\mathrm{H} \\
\mathrm{W}\end{array}$} & \multirow{3}{*}{$\begin{array}{l}\Delta \mathrm{x} \\
\mathrm{mm}\end{array}$} & \multicolumn{2}{|c|}{ A } & \multirow{3}{*}{$\begin{array}{c}k \\
W / m^{0} K\end{array}$} & \multirow{3}{*}{$\begin{array}{c}\text { PROMEDIO } \\
\mathbf{W} / \mathbf{m}^{0} \mathrm{~K}\end{array}$} & \multirow{3}{*}{$\begin{array}{c}\begin{array}{c}\text { Conductividad } \\
\text { según norma }\end{array} \\
\mathrm{W} / \mathrm{m}^{0} \mathrm{~K}\end{array}$} \\
\hline & & & & & & $\mathbf{a}$ & & & & \\
\hline & & & & & & $\mathrm{mm}$ & $\mathrm{mm}$ & & & \\
\hline \multirow{3}{*}{$\begin{array}{l}\text { POLIESTIRENO } \\
\text { EXPANDIDO }\end{array}$} & 1 & 42,2 & 21,2 & 1,02 & 15 & 150 & 150 & 0,032 & \multirow{3}{*}{0,032} & \multirow{3}{*}{0,03} \\
\hline & 2 & 41,2 & 21,2 & 1,02 & 15 & 150 & 150 & 0,033 & & \\
\hline & 3 & 43,1 & 21,3 & 1,03 & 15 & 150 & 150 & 0,031 & & \\
\hline \multirow{3}{*}{$\begin{array}{c}\text { PLACA DE YESO } \\
\text { LAMINADO }\end{array}$} & 1 & 36,7 & 21,1 & 6,02 & 15 & 150 & 150 & 0,257 & \multirow{3}{*}{0,239} & \multirow{3}{*}{0,25} \\
\hline & 2 & 35,1 & 20,4 & 5,06 & 15 & 150 & 150 & 0,229 & & \\
\hline & 3 & 37,4 & 21,6 & 5,47 & 15 & 150 & 150 & 0,231 & & \\
\hline
\end{tabular}




\section{Resultados y recomendaciones}

Es posible la construcción de un aparato de conductividades térmicas de bajo coste y tecnología open source con la suficiente precisión para la investigación de aislantes térmicos naturales alternativos a los aislantes industrializados convencionales.

En climas templados en donde el agua corriente tiene moderadas temperaturas se hace necesario el incremento de la potencia de la placa caliente o la introducción de fluido refrigerado en la placa fría para mantener un diferencial de temperaturas suficiente entre ambas para el cálculo de conductividades en aislamientos de gran espesor.

Pese al correcto funcionamiento, en futuras evoluciones del aparato se hace necesario usar agua refrigerada en el circuito de la placa fría para fomentar una estabilización más rápida e impedir la influencia de las variaciones de T del agua así como su flujo. De esta forma se asegura el estado estacionario de los experimentos

\section{Bibliografía}

J. Diez Campos, E. García Breijo. I Perez Clemente, J.V. Ros Lis, J. Soto Camino (2007). Determinación experimental de la conductividad. XI Congreso Internacional de Ingeniería de Proyectos.

Lopez, R., Morales, J., Diaz, A., \& Lizardi, A. (2000). Equipo para evaluar en forma experimental el coeficiente de conductividad térmica de los materiales de cosntrucción. Revista Mexicana de Física, 47(2), 148-152.

Martínez Fuentes, V. (2004). Sistema primario de medición de conductividad térmica de aislantes térmicos. Etapa 1. Diseño térmico e instrumental. Simposio de Meterorología CENAM

Method, S. T. (2013a). C1114-06. Standard Test Method for Steady-State Thermal Transmission Properties by Means of, $5-12$.

Method, S. T. (2013b). C177-13. Standard Test Method for Steady-State Heat Flux Measurements and Thermal Transmission Properties by Means of the Guarded-Hot-Plate, 1-23.

Method, S. T. (2013c). C518-10. Standard Test Method for Steady-State Thermal Transmission Properties by Means of the Heat Flow Meter Apparatus 1, 1-16.

Method, S. T. (2014). C168-13. Standard Terminology Relating to Thermal Insulation, 1-7. 
Milagrosa, M., Sánchez, P., Centeno, R., \& Lazcano, F. (2002). Desarrollo de un prototipo para la caracterización térmica de los materiales de construcción regionales. Ingeniería, 2, 13-22.

Rodríguez, F. (2007). Diseño, fabricación y calibración de un dispositivo para la caracterización de las propiedades termofísicas en materiales secos de alta resistencia térmica y baja temperatura de uso. Universidad Autónoma de Barcelona.

J. Diez Campos, E. García Breijo. I Perez Clemente, J.V. Ros Lis, J. Soto Camino (2007). DETERMINACIÓN EXPERIMENTAL DE LA CONDUCTIVIDAD. In XI Congreso Internacional de Ingeniería de Proyectos.

Lopez, R., Morales, J., Diaz, A., \& Lizardi, A. (2000). Equipo para evaluar en forma experimental el coeficiente de conductividad térmica de los materiales de cosntrucción. Revista Mexicana de Física, 47(2), 148-152.

Martínez Fuentes, V. (2004). Sistema primario de medición de conductividad térmica de aislantes térmicos. Etapa 1. Diseño térmico e instrumental. Simposio de Meterorología CENAM

Method, S. T. (2013a). C1114-06. Standard Test Method for Steady-State Thermal Transmission Properties by Means of, 5-12.

Method, S. T. (2013b). C177-13. Standard Test Method for Steady-State Heat Flux Measurements and Thermal Transmission Properties by Means of the Guarded-Hot-Plate, 1-23.

Method, S. T. (2013c). C518-10. Standard Test Method for Steady-State Thermal Transmission Properties by Means of the Heat Flow Meter Apparatus 1, 1-16.

Method, S. T. (2014). C168-13. Standard Terminology Relating to Thermal Insulation, 1-7.

Milagrosa, M., Sánchez, P., Centeno, R., \& Lazcano, F. (2002). Desarrollo de un prototipo para la caracterización térmica de los materiales de construcción regionales. Ingeniería, 2, 13-22.

Rodríguez, F. (2007). Diseño, fabricación y calibración de un dispositivo para la caracterización de las propiedades termofísicas en materiales secos de alta resistencia térmica y baja temperatura de uso. Universidad Autónoma de Barcelona. 